\title{
Vulnerabilidad social y la minería en el Perú: un análisis comparativo
}

\author{
MARTHA JHIANNINA CARDENAS* \\ Universidad Federal de Viçosa \\ martha.ruiz@ufv.br \\ MARIA SARAIVA** \\ Universidad Federal de Viçosa \\ mdora@ufv.br \\ https://doi.org/10.18800/rcpg.201602.010
}

\section{RESUMEN}

El presente trabajo tiene como objetivo identificar si la minería implica mayor vulnerabilidad social en Perú. Se realizó una investigación de naturaleza cuantitativa con datos del Instituto Nacional de Estadística e Informática. Como instrumento de análisis se utilizó el programa estadístico SPSS 18.0, y por medio del análisis factorial se asociaron las variables en tres factores que denominamos: Familiar, Físico y Social. Después se utilizó la técnica de cluster, en la cual se agrupó los departamentos con características semejantes. Los resultados mostraron que el cluster 2 , compuesto por una región minera que a su vez tiene presencia de minería ilegal e informal, tiene mayor impacto sobre la vulnerabilidad social, de acuerdo con el test t de Student. Se puede concluir que las regiones mineras influyen en la cantidad de empleos y en la economía local, pero genera vulnerabilidad social, representada por situaciones de denuncias y robos.

Palabras clave: vulnerabilidad social, minería, análisis factorial, análisis cluster, reflexión.

\section{Social vulnerability and mining in Peru: a comparative analysis}

\begin{abstract}
This paper aims to identify if mining implies greater social vulnerability in Peru. A quantitative research was carried out with data from the National Institute of Statistics and Informatics. The statistical program SPSS 18.0 was used as the instrument of analysis by means of factor analysis, in which the variables were associated with three factors: Family, Physical and Social. Then the cluster technique was used, in which the departments with similar characteristics were grouped together. The results showed that the cluster number 2 is composed of a mining region which in turn has illegal and informal mining presence and has a greater impact on

* Graduada en Economía Doméstica y Ciencias Económicas por la Universidad Federal de Vicosa.

** Estudiante de Maestría en la Universidad Federal de Vicosa.

+ Recibido el 16 de junio de 2016; aceptado el 31 de enero de 2017.
\end{abstract}


social vulnerability, according to Student's t test. It can be concluded that the mining regions influence the number of jobs, and the local economy but generates social vulnerability, represented by situations of denunciations and robberies.

Key words: Social vulnerability, Mining, Factor analysis, Cluster analysis Reflection. 


\section{INTRODUCCIÓN}

La actividad minera siempre se constituyó como soporte para la gran industria manufacturera y de joyas del mundo. También es una actividad que está vinculada a las finanzas y al medioambiente. El Perú ocupa uno de los puestos más importantes en América Latina y a nivel mundial por su producción y potencia en minería. En la historia de la economía peruana, la minería siempre contribuyó al crecimiento económico del país y fue parte de una fuente importante de ingresos fiscales. Pero el desarrollo de conflictos e impactos ambientales siempre fue motivo de preocupación para las comunidades rurales y la sociedad en general.

En el Anuario Minero 2014 elaborado por el Ministerio de Energía y Minas (2015), se afirma que la inversión en el país trajo consigo crecimiento económico en cada región y también desarrolló una minería más sostenible. Sobre esta afirmación, otros autores opinan lo contrario, Carrere (2004), por ejemplo, considera que la actividad minera es necesaria para satisfacer de bienes a los seres humanos, pero no se puede argumentar que esta actividad sea sostenible, pues es una actividad que extrae recursos no renovables.

La inversión en minería trajo consigo el aumento de empleo en las economías locales, como lo afirma el Ministerio de Energía y Minas. Otros autores, como Macdonald y Rowland (2002), afirmaron los cambios de los estilos de vida y empleo, pues antes se hacían actividades de agricultura que involucraban a toda la familia.

El objetivo de este estudio es identificar si las regiones mineras poseen mayor vulnerabilidad social. La hipótesis es que la presencia de actividades mineras tiende a impactar negativamente la vulnerabilidad. Para este análisis se utilizó la técnica de cluster para agrupar las regiones mineras que tengan una semejanza y se aplicó un test paramétrico de muestras independientes (t de Student) para verificar el impacto de las regiones mineras en la vulnerabilidad social.

\section{FundAMENTOS TEÓRICOS}

La minería peruana tiene su inicio en la época preinca, y a lo largo del tiempo tuvo una evolución que hoy en día representa, según el Estado, una actividad productiva moderna y responsable, además que constituye una de las bases estratégicas de desarrollo y crecimiento económico en el país. En la historia de la economía peruana, se destaca que la minería determina el crecimiento económico del país, pero los impactos ambientales generados por esta 
actividad afectaron el desarrollo de la minería (Lira y Aristondo, 2007). En el Cuadro 1 podemos observar la posición de Perú en el ranking de minería a nivel mundial.

Cuadro 1. Posición de Perú en el ranking mundial de la producción en minería, 2014

\begin{tabular}{|l|c|c|}
\hline Producto & Latinoamérica & Mundo \\
\hline Zinc & 1 & 3 \\
\hline Estaño & 1 & 4 \\
\hline Plomo & 1 & 7 \\
\hline Oro & 1 & 3 \\
\hline Cobre & 2 & 3 \\
\hline Plata & 2 & 4 \\
\hline Molibdeno & 2 & 9 \\
\hline Selenio & 2 & 8 \\
\hline Cadmio & 2 & 12 \\
\hline Roca fosfórica & 2 & 3 \\
\hline
\end{tabular}

Fuente: Ministerio de Energía y Minas (2015).

La cordillera de los Andes siempre fue una fuente de recursos minerales para el Perú. Como se ha mencionado anteriormente, la minería genera valor agregado, divisas, inversión y empleo. Asimismo, se puede ver la importancia de la minería a nivel departamental por la participación en esta actividad económica. La minería representa el 9,70\% del PBI y el 57,11\% de las exportaciones peruanas. El PBI minero juega un rol importante en la economía del país, pero en 2014 tuvo un decrecimiento debido a la falta de leyes y acuerdos con algunas empresas mineras. En el Cuadro 2 se puede observar la evolución entre los ańos 2012 y 2014 para el Perú del PBI y del PBI minero, nivel de exportaciones e importaciones y exportaciones mineras de acuerdo con el Ministerio de Energía y Minas (2015).

Para diferenciar las regiones mineras de las no mineras se utiliza el cálculo agregado, donde se considera mineras a aquellas regiones cuya incidencia del VAB minero sobre el VAB total supera el $20 \%$ en el respectivo año, según el Instituto Peruano de Economía. De esta manera, las regiones mineras son las siguientes: Áncash, Apurímac, Arequipa, Cajamarca, Cusco, Huancavelica, Loreto, Madre de Dios, Moquegua, Pasco y Tacna. 
Cuadro 2. Principales variables macroeconómicas del Perú (2012-2014)

\begin{tabular}{|l|c|c|c|}
\hline Producto & 2012 & $\mathbf{2 0 1 3}$ & $\mathbf{2 0 1 4}$ \\
\hline PBI (var \% real) & $5,95 \%$ & $5,77 \%$ & $2,35 \%$ \\
\hline PBI minero (var \% real) & $2,51 \%$ & $4,26 \%$ & $-2,11 \%$ \\
\hline Inflación tasa \% & $2,65 \%$ & $2,86 \%$ & $3,22 \%$ \\
\hline $\begin{array}{l}\text { Tipo de cambio promedio } \\
\text { (S/. por US\$) }\end{array}$ & 2,64 & 2,7 & 2,84 \\
\hline Exportaciones (US\$ MM) & 47411 & 42474 & 39326 \\
\hline $\begin{array}{l}\text { Exportaciones mineras } \\
\text { (US\$ MM) }\end{array}$ & 27467 & 23554 & 20410 \\
\hline $\begin{array}{l}\text { Exportaciones mineras } \\
\text { (US\$ MM) }\end{array}$ & 41135 & 42217 & 40807 \\
\hline
\end{tabular}

Fuente: Ministerio de Energía y Minas (2015).

\section{Cuadro 3. Regiones mineras y el VAB minero}

\begin{tabular}{|l|c|c|}
\hline \multirow{2}{*}{ Departamento } & $\begin{array}{c}\text { VAB } \\
\text { (Variación porcentual } \\
\text { anual) }\end{array}$ & $\begin{array}{c}\text { Mundo del total valor agregado bruto } \\
(\mathbf{1 0 0} \%)\end{array}$ \\
\cline { 3 - 3 } & $-12,2$ & $\begin{array}{c}\text { Estructura porcentual } \% \\
\text { Extracción de petróleo, gas y minerales }\end{array}$ \\
\hline Áncash & 4,6 & $55,0 \%$ \\
\hline Apurímac & 0,8 & $28,5 \%$ \\
\hline Arequipa & $-0,9$ & $26,7 \%$ \\
\hline Cajamarca & 0,5 & $30,5 \%$ \\
\hline Cusco & 4,1 & $33,6 \%$ \\
\hline Huancavelica & 3,3 & $26,3 \%$ \\
\hline Loreto & $-13,5$ & $35,1 \%$ \\
\hline Madre de Dios & $-2,6$ & $53,0 \%$ \\
\hline Moquegua & 3,1 & $39,7 \%$ \\
\hline Pasco & 5,7 & $76,4 \%$ \\
\hline Tacna & & $48,5 \%$ \\
\hline
\end{tabular}

${ }^{a}$ Corresponde a la variación porcentual del índice de volumen físico.

${ }^{\text {b }}$ Estructura porcentual del año base 2007.

Fuente: Instituto Nacional de Estadística e Informática (2015). 
Perú es un país minero debido a sus yacimientos y la diversidad de los mismos. Asimismo, los proyectos que se generarían, identificados por el Ministerio de Energía y Minas, superan los US\$ 60000 millones de inversión, los cuales, según Duran (2014), llevarían a un incremento en las exportaciones, aumento de puestos de trabajo directos e indirectos, y generación de impuestos indirectos. De esa manera, el empleo en minería genera nueve empleos adicionales en otros sectores. Se concluye que esta actividad es vital para la economía peruana, así como para su producción, empleo e ingresos fiscales (Duran, 2014).

La nueva minería en el Perú se expande desde zonas tradicionales altoandinas hasta nuevas regiones, que son valles agrícolas de la costa y de la región quechua. Hasta finales del siglo XIX, la minería demandaba insumos para producción y bienes de consumo para los campamentos de minería, con repercusiones en la formación del mercado laboral local. Asimismo, a inicios del siglo $\mathrm{XX}$, los trabajadores andinos que ejercían actividades extractivas mantenían una condición mixta entre campesino y operario minero. Con la llegada del capital norteamericano durante el siglo XX, se crea un mercado laboral asalariado que tiene influencia en la monetización de las economías regionales de la sierra (Contreras, 1988). Se destaca que estas actividades traen consigo cambios en el estilo de vida de las personas, como la competencia entre las actividades de minería y agricultura, como muestra el estudio de Méndez, Orihuela y Paredes (2007) en los distritos de la sierra (norte, centro y sur), cuando los ingresos en las zonas rurales obtuvieron un aumento producto de la minería; sin embargo, los autores mencionados afirman también que para las familias urbanas de la sierra sur se observa un aumento de la pobreza con la presencia de la minería.

En diciembre de 2014, la Defensoría del Pueblo reportó 210 conflictos sociales (160 activos y 50 latentes), todos asociados a la explotación de los recursos minerales. Estos conflictos fueron por reclamaciones por asuntos de contaminación del agua y tierra, ventas de propiedades no legítimas o injustas, pasivos ambientales de operaciones pasadas, demandas por mayores transferencias de recursos (la comunidad, el distrito y la región) y, finalmente, la oposición a iniciar nuevos proyectos de explotación de la producción.

Carrere (2004) describe los impactos sociales y ambientales de la minería, y considera que la minería es responsable por la pérdida del sustento de millones de personas, de guerras civiles, dictaduras e intervenciones armadas extranjeras, también por la violación de los derechos humanos, envenenamiento de personas y del medioambiente, así como es una de las causas de la deforestación y degradación de los bosques. Por otro lado, describe la dependencia de las 
exportaciones de minerales de los países de América del Sur y su estándar de vida. Así, los niveles más altos de dependencia de los minerales se correlacionan con mayores niveles de pobreza y tasas de desnutrición y mortalidad infantil. También, el autor mencionado nos revela que se tiende a sufrir tasas de corrupción, de gobiernos autoritarios, ineficacia gubernamental y gastos militares.

En este sentido, es importante destacar que la minería puede traer consigo impactos negativos en la vida de la personas que viven cerca de las zonas donde se desarrollan estas actividades, donde se substituyen las economías de subsistencia que alimentan a generaciones de comunidades y pueblos indígenas por una economía basada en el dinero en efectivo. Esta nueva economía implica una erosión y destrucción de valores y costumbres tradicionales que siempre fueron esenciales para el sustento de la solidaridad y la unidad de familias, tribus y comunidades. En estas situaciones, la mujer es la más marginada, debido a cambios en los papeles tradicionales, que antes era de recolección de alimentos, administración del agua y cuidadora. También es necesario comprender que las compañías habitualmente entran en contacto y hacen negociaciones con hombres, excluyendo a las mujeres de pagos de compensaciones. Incluso tienen poco o nada de control con respecto a los beneficios de la explotación de las minas, casi no acceden al dinero ni menos a empleos. De este modo, son más dependientes de los hombres, que tienen más probabilidad de acceder a este tipo de beneficios.

Así como la minería a gran escala brinda pocas oportunidades de empleo a la mujer, el sector de menor escala absorbe a las mujeres como trabajadoras temporales o en trabajos forzosos, muchas veces en condiciones de explotación severa. Por todo eso, las mujeres muchas veces están expuestas a la explotación física y sexual por parte de los dueños o trabajadores de las minas y de los comerciantes locales que venden los minerales. Como destacan Macdonald y Rowland (2002), es evidente que el abuso del alcohol, la dependencia de las drogas, la prostitución, las apuestas, el incesto y la infidelidad tienen un aumento en las comunidades donde está presente la minería. Se registran casos de violencia familiar contra las mujeres, discriminación activa en el lugar de trabajo de ellas, que frecuentemente son sancionadas o ignoradas por las instituciones judiciales y políticas. En resumen, la minería, sea en pequeña o gran escala, produce impactos específicos en la mujer, así como en las familias, y se está perdiendo el control en los aspectos relacionados con el desenvolvimiento de esta actividad.

En la última década el volumen de conflictos sociales fue aumentando, y se vincula de forma directa o indirecta a las actividades de minería, pese a que 
se cree que esta es un motor de desarrollo de actitudes favorables, lo que no es compartido por los pobladores que viven alrededor de las zonas de explotación minera (Glave y Kuramoto, 2007; Bebbington y Humphreys, 2009). Los pobladores sufren de las siguientes consecuencias a raíz de la actividad minera: los riesgos ambientales de la minería, la modificación y hasta la destrucción de las formas de vida y organización social de las comunidades rurales andinas, amazónicas e inclusive de la costa. Las personas que viven cerca de las zonas de actividad minera están expuestas a consecuencias ambientales (contaminación, perjuicio para agricultura y pesca), sociales (destrucción de las formas de vida originales y aparición de actividades delictivas) y económicas (pocas expectativas con respecto al progreso económico social) (De Echave, Diez, Huber, Revesz, Lanata y Tanaka, 2009).

\section{Vulnerabilidad social}

En el mundo actual se puede observar que la vulnerabilidad social es un fenómeno muy frecuente, sobre el tema se destacan estudios donde la ausencia de servicios y las condiciones de vida adecuadas, la falta de empleo y el ocio son las principales motivaciones para generar actos de violencia. La vulnerabilidad social es vista como un resultado negativo dentro de la relación entre la disponibilidad de recursos de los actores y el acceso a oportunidades sociales, económicas y culturales provenientes del Estado, mercado y sociedad civil.

Kaztman (2000) describe un enfoque de vulnerabilidad referido al estado de las familias, que tiene una variación inversa con las capacidades de las familias de controlar las fuerzas que hacen su propio destino, o para restar sus efectos sobre el bienestar. La noción de vulnerabilidad está centrada en situaciones en las que tiene que haber un acceso a las oportunidades que brinda el Estado, el mercado y la sociedad. Las distintas inadaptaciones, que son resultado de las estructuras de oportunidades de mercado, traen como consecuencia un aumento de situaciones de precariedad e inestabilidad laboral. Los distintos desfases con respecto a la oportunidad del Estado y la sociedad, resultan en un aumento de las situaciones de desprotección e inseguridad. Para medir la vulnerabilidad se requiere datos sobre activos de las familias (físicos, humanos y de capital) en combinación con datos sobre redes formales de seguridad, así como sobre el funcionamiento del mercado y políticas económicas que determinen las oportunidades laborales de las familias.

Según Busso (2001), la vulnerabilidad puede ser entendida «como un proceso multidimensional que confluye en el riesgo o la probabilidad de que el individuo, familia o comunidad de ser herido, lesionado contra el cambio o 
la permanencia de situaciones externas o internas» (p. 8). La vulnerabilidad social, ya sea de un individuo o comunidad, está expresado en tres formas: fragilidad, desamparo y debilidad interna para afrontar los cambios necesarios de un individuo o familia para las oportunidades. La noción de vulnerabilidad se asocia con la expresión «a qué» se es vulnerable. El uso de estos adjetivos tiene una relación con enfoques sobre temas económicos, ambientales, desastres naturales y con la salud física y mental de los individuos. Pero en los últimos años se realizaron estudios sobre vulnerabilidad social, psicosocial, jurídica, política, demográfica, entre otras áreas. Por lo tanto, vulnerabilidad es una noción multidimensional en la medida que afecta el bienestar de individuos, grupos y comunidades, de diversas formas e intensidades. Se puede decir que los seres humanos, y también las comunidades, son en mayor o menor medida vulnerables, según la renta, patrimonio, lugar de residencia, país de nacimiento, origen étnico, género, incapacidad, enfermedades, factores políticos, ambientales o diversos motivos que de alguna u otra forma los exponen a riesgos. Busso (p. 9) concluye que la noción de vulnerabilidad puede ser entendida como un proceso que relaciona diversos niveles de análisis y puede existir una sinergia negativa entre distintas dimensiones en la medida en que puede generar incrementos de los niveles de vulnerabilidad a partir de una situación de vulnerabilidad inicial, produciendo un círculo vicioso de la vulnerabilidad entre las distintas unidades de análisis y las diversas dimensiones de la misma.

Cuando se habla de vulnerabilidad social, se refiere a la predisposición en la que un grupo es afectado, en términos físicos, económicos, políticos y sociales, en caso de ocurrencia de un proceso o acción de origen natural o antrópico. La atención política que se tiene sobre la reducción de la vulnerabilidad está más centrada en la reducción de los costos y en la medida técnica y operacional de la vulnerabilidad que en las propias cuestiones de ciudadanía, calidad de vida y seguridad estructural de las poblaciones (Mendes y Tavares, 2011).

Según Cunha (2004), el concepto de vulnerabilidad trae consigo dieciocho tipos de definición de vulnerabilidad. En la visión del autor, el enfoque de vulnerabilidad en su estudio está inclinado a la aprehensión de factores que convierten a los individuos y familias en más o menos vulnerables, sin olvidar el concepto en relación con el territorio. Se habla sobre la vulnerabilidad de personas y no de recursos naturales, donde se incluye el papel de procesos sociodemográficos en el direccionamiento del vínculo de esos factores. El estudio investiga los procesos sociodemográficos, urbanos y ambientales, que derivan del fenómeno metropolitano, en las regiones metropolitanas de Campinas y Santos localizadas en el estado de Sáo Paulo. 
Por otro lado, Torralba (1998) dice que todo ser humano es vulnerable en todas sus dimensiones, es decir, es vulnerable en el sentido físico porque el sujeto está expuesto a quedar enfermo y sufrir de alguna incapacidad, también es vulnerable psicológicamente porque su mente es frágil, asimismo es vulnerable socialmente, pues como agente social es susceptible de tensiones e injusticia social, y finalmente el sujeto también puede ser vulnerable espiritualmente. Denomina «vulnerabilidad natural» al medioambiente del ser humano que siempre está expuesto a cambios y transformaciones. La naturaleza es frágil, por eso se debe cuidar y evitar el deterioro de la realidad natural, pues esto afecta a la estructura personal y la forma de vivir, trabajar y hasta de amar. La vulnerabilidad social está referida a la socialización del ser humano. Las relaciones interpersonales del sujeto se desenvuelven en el plano de la amistad, el amor y el respeto, asimismo puede desenvolver el plano de la violencia y la instrumentalización. Por lo tanto, la vulnerabilidad social del individuo puede expresar la violencia en la sociedad con la pérdida de la seguridad social.

Así, una de las manifestaciones de la vulnerabilidad social es la violencia, cuyo concepto básico siempre está en constante cambio; es decir, no es fácil definirla porque, muchas veces, puede dejar fuera a las víctimas y no tomar en cuenta las "pequeñas violencias» que son realizadas de forma cotidiana tales como comportamientos que conllevan a la discriminación y el ejercicio del poder como la expresión de gestos, frases cortas, silencios acusatorios que vuelven invisible la discriminación y el maltrato, además son comunes en el ambiente laboral, educativo y en los medios de comunicación social, cuando denigra la imagen de las personas y se transmiten mensajes discriminatorios. La violencia muchas veces está asociada a la pobreza, no es una consecuencia directa, pero sí la forma de negación del derecho al acceso de alimentos y bienes, el ocio, el deporte y la cultura; la desigualdad social también opera en cada grupo social, trayendo consigo comportamientos violentos (Abramovay y Pinheiro, 2003). Según Abramovay y Pinheiro la violencia puede ser de dos tipos:

A violência se refere no sentido estrito à violência física como uma intervenção por parte de uma pessoa ou grupo contra a integridade de outros individuo se também contra si mesmo. Tal definição abarca desde os suicídios, espaçamentos de vários tipos, robôs, assaltos e homicídios ate a violência no transito (camuflada sobre o nome de "acidentes") e todas as diversas formas de agressão sexual...Já a violência simbólica se refere ao abuso do poder baseado no consentimento que se estabelece e se impóe mediante o uso de símbolos de autoridade, como violência verbal e também a violência institucional marginalização, discriminação e praticas de assujeitamento utilizadas por instituiçôes diversas que instrumentalizam estratégias de poder (p. 2). 
En este contexto, Pizarro (2001) afirma que el concepto de vulnerabilidad social tiene dos componentes: el primero es la inseguridad y desprotección que experimentan las familias, comunidades e individuos en sus condiciones de vida a consecuencia del impacto provocado por un evento económico-social, que tiene carácter traumático, así como por los desastres naturales. El otro componente se enfoca en los cambios de las condiciones de vida que experimentan las comunidades rurales pobres en el contexto de eventos socioeconómicos traumáticos.

León (2006) define la vulnerabilidad de acuerdo al ISDR (International Strategy for Disaster Reduction) como un «conjunto de condiciones y procesos que son resultados de factores físicos, sociales, económicos y ambientales que aumentan la susceptibilidad de una comunidad a los impactos y riesgos» (p. 13). Los factores físicos están constituidos por la localización y el ambiente construido, o también puede ser representado por la densidad de la población, la eliminación de un establecimiento local, de las materias de construcción y técnicas empleadas para la construcción de la infraestructura. Los factores sociales están relacionados a niveles de bienestar de los individuos con respecto a salud, alfabetización, educación, la existencia de paz y seguridad, el acceso a los derechos humanos, la justicia social, valores tradicionales, creencias y sistemas organizacionales. En enfoques económicos, están relacionados a las cuestiones de pobreza, que incluye las reservas económicas nacionales, los niveles de deuda, grados de acceso al crédito, préstamos y seguros, y diversidad económica. Finalmente, en los factores ambientales se incluye la disminución de los recursos naturales y la degradación. Algunos de los elementos que pueden influenciar la vulnerabilidad ambiental son la exposición a contaminantes tóxicos y peligrosos, acceso reducido al aire limpio, agua y saneamiento, y las formas inadecuadas de gestión de residuos (León, 2006).

\section{Metodología}

Los antecedentes en estudios sobre minería y sus diferentes impactos en la sociedad son los siguientes: de naturaleza cuantitativa tenemos a Orihuela, Huaroto y Paredes (2014), que nos afirman que a mayor actividad minera (antigua, actual y futura), se genera mayor conflicto; asimismo, por medio de la econometría se encontró que la minería no genera beneficios tangibles para la actividad agropecuaria. Zegarra, Orihuela y Paredes (2007) también hicieron una investigación de naturaleza cuantitativa, donde se estudió el impacto de la actividad minerametálica en los hogares de la sierra peruana. Barrantes, Zárate y Durand (2005) 
analizaron la relación entre la actividad minera, el desarrollo y la pobreza; primero examinaron la contribución del sector minero a las cuentas macroeconómicas del país para saber los beneficios de esta actividad en la economía peruana, después analizaron la importancia del canon en las cuentas municipales y cómo se reparte desde el Gobierno central, y por último examinaron los ingresos de los hogares que pertenecen a distritos mineros para ver cuán pobres eran y compararlos con otros hogares que no tienen presencia de minería.

Arellano (2011) analiza la política peruana y examina la relación entre el conflicto social y la Nueva Estrategia de las Industrias Extractivas (NEIE) en las regiones mineras peruanas. Para obtener sus resultados hace un análisis cuantitativo de los factores que explican las variaciones en la incidencia de conflictos sociales en las distintas regiones peruanas. Asimismo, concluye que existe una correlación entre el incremento de conflictos y la cantidad de canon minero que reciben los Gobiernos regionales. Loayza, Mier y Terán y Rigolini (2013) hicieron un estudio sobre la pobreza, desigualdad y la maldición local de los recursos naturales, donde comunidades locales son beneficiadas por los auges de productos básicos. Investigan el impacto local sobre los resultados socioeconómicos de la actividad minera en el Perú, donde utilizan una investigación cuantitativa que da como resultado que los distritos productores tienen mejores niveles de vida en comparación con distritos que no son productores con respecto al mayor consumo de los hogares, menor tasa de pobreza y mayor alfabetización. Sin embargo, esos beneficios son disminuidos significativamente con la distancia administrativa y geográfica de la mina.

El presente estudio se basó en datos estadísticos del Perú, que tiene veinticuatro departamentos y una provincia constitucional, el Callao. Para este estudio se consideró regiones mineras del año 2014. Por tanto, fueron once departamentos los estudiados. Las variables utilizadas están descritas en el Cuadro 4.

\subsection{Análisis factorial}

Para este estudio se ha utilizado el análisis factorial, que es una técnica de interdependencia cuyo propósito es definir la estructura intrínseca entre las variables en análisis. El análisis factorial se caracteriza por proporcionar las herramientas para llevar una revisión de la estructura de interrelaciones (correlaciones) en un gran número de variables, que se definen como un conjunto de variables que están fuertemente interrelacionadas, conocido como factores. Estos grupos de variables (factores), que están correlacionados, son considerados como representantes de dimensiones dentro de los datos. En el análisis factorial importa más el carácter y la composición de las variables. 


\section{Cuadro 4. Descripción de las variables de estudio}

\begin{tabular}{|c|c|}
\hline Variables & Concepto y descripción \\
\hline $\begin{array}{l}\text { Valor agregado bruto } \\
\text { minero }\end{array}$ & $\begin{array}{l}\text { Valor agregado bruto minero según departamento (precios constantes, } \\
\text { ańo base 2007). }\end{array}$ \\
\hline Denuncias de faltas & $\begin{array}{l}\text { Casos registrados de denuncias de faltas contra la persona, como: lesión } \\
\text { culposa, maltrato y agresión sin daño, contra el patrimonio, contra las } \\
\text { buenas costumbres, entre otras. Calculado sobre la razón de la población } \\
\text { existente por departamento. }\end{array}$ \\
\hline Denuncias de delitos & $\begin{array}{l}\text { Casos registrados de delitos cometidos por departamento en } 2014 \text {. Estos } \\
\text { son delitos contra la vida, cuerpo y salud, contra la familia, libertad, } \\
\text { patrimonio, orden público, orden financiero, tributarios, fe pública, } \\
\text { seguridad pública, tranquilidad pública, humanidad, agravados, contra la } \\
\text { administración pública, posesión de armas, entre otros. Calculado sobre } \\
\text { la razón de la población existente por departamento. }\end{array}$ \\
\hline $\begin{array}{l}\text { Denuncias de violencia } \\
\text { familiar }\end{array}$ & $\begin{array}{l}\text { Casos registrados de violencia familiar por agresión física y psicológica } \\
\text { según departamento. Calculado sobre la razón de la población existente } \\
\text { por departamento. }\end{array}$ \\
\hline $\begin{array}{l}\text { Personas que fueron } \\
\text { intervenidas por tráfico } \\
\text { ilícito de drogas }\end{array}$ & $\begin{array}{l}\text { Número de personas por departamento que fueron intervenidas en } \\
\text { tráfico ilícito de drogas en } 2014 \text {. Calculado sobre la razón de la población } \\
\text { existente por departamento. }\end{array}$ \\
\hline $\begin{array}{l}\text { Personas que fueron } \\
\text { intervenidas por consumo } \\
\text { de drogas }\end{array}$ & $\begin{array}{l}\text { Personas por departamento que fueron intervenidas por consumo de } \\
\text { drogas en el año } 2014 \text {. Calculado sobre la razón de la población existente } \\
\text { por departamento. }\end{array}$ \\
\hline $\begin{array}{l}\text { Denuncias por robo de } \\
\text { vehículos }\end{array}$ & $\begin{array}{l}\text { Casos registrados de robo de vehículos por departamento. Calculado } \\
\text { sobre la razón de la población existente por departamento. }\end{array}$ \\
\hline $\begin{array}{l}\text { Denuncias de accidentes } \\
\text { de tránsito no fatales }\end{array}$ & $\begin{array}{l}\text { Casos registrados de accidentes de tránsito no fatales. Calculado sobre la } \\
\text { razón de la población existente por departamento. }\end{array}$ \\
\hline $\begin{array}{l}\text { Casos de víctimas de } \\
\text { feminicidio }\end{array}$ & $\begin{array}{l}\text { Casos registrados por feminicidios según departamentos. Calculado } \\
\text { sobre la razón de la población existente por departamento. }\end{array}$ \\
\hline $\begin{array}{l}\text { Casos de violación sexual a } \\
\text { menores de } 18 \text { años }\end{array}$ & $\begin{array}{l}\text { Denuncias de violencia sexual en personas menores de } 18 \text { años de edad } \\
\text { según departamento. Calculado sobre la razón de la población existente } \\
\text { por departamento. }\end{array}$ \\
\hline
\end{tabular}

Fuente: Datos obtenidos por el Instituto Nacional de Estadística e Informática-INEI.

Los supuestos del análisis de factores son los siguientes: el básico es que subyace en el conjunto de variables elegidas, que establece que el investigador asegure que los patrones observados son conceptualmente válidos y apropiados para estudiar con el análisis factorial; con respecto a la técnica, no tiene ningún medio para determinar la idoneidad más allá de las correlaciones entre las variables. Otra hipótesis es que tiene la presencia de multicolinealidad que es 
deseable, ya que el objetivo es identificar conjuntos de variables interrelacionadas. $\mathrm{O}$ sea, el investigador debe asegurarse de que la matriz de datos tiene suficientes correlaciones para justificar el análisis factorial.

De acuerdo con Hair, Black, Babin, Anderson y Tatham (2009), una prueba de significación estadística de esfericidad de Bartlett (sig. <0,05) indica que existen suficientes correlaciones entre las variables para continuar con el análisis. Las medidas de valores de adecuación para la muestra (MAS) tiene que exceder 0,5 tanto para el test general como para cada variable individual, y las variables inferiores a 0,05 deben ser omitidas del análisis factorial.

\subsection{Análisis cluster}

La segunda técnica usada es el análisis de cluster o de agrupamiento. Esta técnica multivariante también se conoce como conglomerados. Su propósito es agrupar objetos que forman conglomerados (clusters) de objetos con un alto grado de homogeneidad interna y heterogeneidad externa. Es decir, las unidades de análisis se clasifican en grupos homogéneos de modo que las unidades que pertenecen a uno de estos grupos o conglomerados son similares entre sí, a pesar de que son muy diferentes respecto a otros grupos. A diferencia del análisis factorial, el análisis de cluster es una técnica de agrupamiento, se puede identificar una amplia variedad de métodos en este análisis de conglomerados. Las dos principales categorías de los métodos estructurados son jerárquicos y no jerárquicos. Este análisis no distingue entre variables dependientes e independientes, pero puede examinar interrelación entre todo el conjunto de variables. Estas consideraciones se deben tener en cuenta para la selección de variables: esta técnica de análisis se puede aplicar sobre variables con cualquier valor de medida, pero es conveniente que todas tengan el mismo valor de medida. Las variables seleccionadas deben tener la misma escala, pues los resultados opuestos pueden verse afectados (Rodríguez y Mora, 2001).

\subsection{Test paramétrico para muestras independientes}

Barbetta (2012) dice que el planeamiento de la investigación debe permitir la prueba de la veracidad de las ideas de los datos de población de estudio. La población es el mundo real y las ideas son las hipótesis de investigación que pueden ser probados para la prueba estadística de hipótesis llamada técnica o pruebas de significación. La aplicación de una prueba estadística (o prueba de significación) sirve para verificar que los datos proporcionan evidencia 
suficiente, de manera que podemos aceptar como cierta la hipótesis de investigación, de esta manera ser precavidos, y tener un cierto grado de certeza, donde las diferencias observadas en los datos no sean meramente casuales (Manteiga y Vargas, 2009).

\section{RESULTADOS Y DisCUSIÓN}

La primera técnica en este estudio fue el análisis factorial, que muestra los resultados a escalas utilizadas para medición de los constructos necesarios para el test de la hipótesis general en este estudio. Por lo tanto, se hizo uso de la rotación Varimax, que es el método de rotación ortogonal que minimiza el número de variables que tiene saturaciones altas en cada factor; asimismo, simplifica la interpretación de los factores optimizando la solución por columna. De acuerdo con Hair et al. (2009), el índice KMO debe estar encima de 0,05; el test Barlett con índice alto y significancia menor que 0,05; y cargas factoriales encima de 0,5 . La Tabla 1 presenta los factores encontrados.

Tabla 1. Descripción de las variables de estudio

\begin{tabular}{|l|c|c|c|}
\hline \multirow{2}{*}{ Variables } & \multicolumn{2}{|c|}{ Factores } \\
\cline { 2 - 4 } & 1. Familiar & 2. Físico & 3. Social \\
\hline Denuncias de faltas & & & 0,677 \\
\hline Denuncias de delitos & 0,751 & & \\
\hline Denuncias por violencia familiar & 0,763 & & \\
\hline $\begin{array}{l}\text { Personas que fueron intervenidas por } \\
\text { tráfico ilícito de drogas }\end{array}$ & 0,825 & & \\
\hline $\begin{array}{l}\text { Personas que fueron intervenidas por } \\
\text { consumo de drogas }\end{array}$ & 0,886 & & \\
\hline Denuncias por robo de vehículos & & & \\
\hline $\begin{array}{l}\text { Denuncias de accidentes de tránsito no } \\
\text { fatales }\end{array}$ & 0,776 & & \\
\hline Victimas de feminicidios & & 0,950 & \\
\hline Violación sexual a menores de 18 ańos & & 0,896 & \\
\hline
\end{tabular}

Nota: Método de extracción: Análisis Principal Componentes.

Método de Rotación: Varimax con Normalización de Kaiser.

Elaboración propia

Fuente: Instituto Nacional de Estadística e Informática (2015). 
Los resultados encontrados confirman la elaboración de tres factores: el factor 1 está compuesto por las variables: denuncias de delitos, denuncias por violencia familiar, personas que fueron intervenidas por tráfico ilícito de drogas y consumo, y denuncias de accidentes de tránsito no fatales, en el cual se formó una dimensión «Familiar». El factor 2 fue constituido por las variables: víctimas de feminicidios y víctimas de violación sexual a menores de 18 años, que fue denominado dimensión "Física». El factor 3 fue formado por las variables: denuncias por faltas y denuncias de robo de vehículos, que fue llamada «Social».

Se utilizó el análisis cluster para agrupar los departamentos más similares posibles, de tal forma que se puedan investigar las regiones mineras. El método Ward se utilizó para minimizar la varianza intergrupal y maximizar la homogeneidad dentro de los grupos. Mediante el programa SPSS, fueron hechos dos grupos, donde el primero permanece con diez observaciones y el segundo con uno, descritas en la Tabla 2.

Tabla 2. Regiones mineras según los clusters

\begin{tabular}{|c|l|c|c|}
\hline Cluster/Grupos & Departamentos & $\begin{array}{c}\text { Valor agregado bruto } \\
\text { minero (año base 2007) }\end{array}$ & $\begin{array}{c}\text { \% VAB minero del } \\
\text { VAB total }\end{array}$ \\
\hline 1 & Áncash & 7018938 & $55,0 \%$ \\
\hline 1 & Apurímac & 113566 & $28,5 \%$ \\
\hline 1 & Arequipa & 4716249 & $26,7 \%$ \\
\hline 1 & Cajamarca & 2717363 & $30,5 \%$ \\
\hline 1 & Cusco & 9899816 & $33,6 \%$ \\
\hline 1 & Huancavelica & 663355 & $26,3 \%$ \\
\hline 1 & Loreto & 2397002 & $35,1 \%$ \\
\hline 2 & Madre de Dios & 614963 & $53,0 \%$ \\
\hline 1 & Moquegua & 2699586 & $39,7 \%$ \\
\hline 1 & Pasco & 3138142 & $76,4 \%$ \\
\hline 1 & Tacna & 2184944 & $48,5 \%$ \\
\hline
\end{tabular}

Fuente: Instituto Nacional de Estadística e Informática (2015).

En el cluster 1, compuesto por diez departamentos que son considerados regiones mineras, su valor agregado bruto minero sobre el valor agregado total supera en $20 \%$. De esta forma, las regiones que se agrupan en el cluster 1 son: Áncash, Apurímac, Arequipa, Cajamarca, Cusco, Huancavelica, Loreto, Moquegua, Pasco y Tacna. 
Por otro lado, el cluster 2 está compuesto por el departamento de Madre de Dios, uno de los departamentos que tiene mayor biodiversidad pero presenta problemas asociados a la contaminación del medioambiente, que es generada por la presencia de la minería informal e ilegal. Según la Policía Nacional del Perú, en esta región se cometen 20,1 homicidios por cada 1000 habitantes, debido a las zonas de minería ilegal. Asimismo, se afirma que en esta región se ha generado una corriente delictiva alarmante debido a la minería ilegal, y esa sería una razón por la que la región no se agrupó con las demás.

Para examinar la asociación de la vulnerabilidad con los dos cluster de regiones mineras se hizo un test $\mathrm{t}$ Student para muestras independientes. La Tabla 3 nos presenta los resultados de los factores probados.

Tabla 3. Resultados test $\mathrm{t}$-Student

\begin{tabular}{|c|c|c|c|c|c|c|c|}
\hline \multirow[t]{2}{*}{ Factor } & \multirow[t]{2}{*}{ Cluster } & \multirow[t]{2}{*}{$\begin{array}{l}\text { Media factor } \\
\text { padronizado }\end{array}$} & \multirow[t]{2}{*}{$\begin{array}{l}\text { Diferencia } \\
\text { de Medias }\end{array}$} & \multicolumn{2}{|c|}{$\begin{array}{l}\text { Intervalo Confidencial } \\
\text { para diferencias de } \\
\text { medias }(95 \%)\end{array}$} & \multirow[t]{2}{*}{$\begin{array}{c}\text { Valor } \\
\text { t-Student }{ }^{\mathrm{a}}\end{array}$} & \multirow[t]{2}{*}{ Sig } \\
\hline & & & & Superior & Inferior & & \\
\hline \multirow{2}{*}{ Familiar } & 1 & $-0,0673$ & \multirow{2}{*}{$-0,7404$} & \multirow{2}{*}{1,6973} & \multirow{2}{*}{$-3,1782$} & \multirow{2}{*}{$-0,687$} & \multirow{2}{*}{0,509} \\
\hline & 2 & 0,6731 & & & & & \\
\hline \multirow{2}{*}{ Físico } & 1 & 0,0210 & \multirow{2}{*}{0,2320} & \multirow{2}{*}{2,7268} & \multirow{2}{*}{$-2,2627$} & \multirow{2}{*}{0,210} & \multirow{2}{*}{0,838} \\
\hline & 2 & $-0,2109$ & & & & & \\
\hline \multirow{2}{*}{ Social } & 1 & $-0,2777$ & \multirow{2}{*}{$-3,0555$} & \multirow{2}{*}{$-2,0828$} & \multirow{2}{*}{$-4,0281$} & \multirow{2}{*}{$-7,106$} & \multirow{2}{*}{0,00} \\
\hline & & 2,7777 & & & & & \\
\hline
\end{tabular}

${ }^{a}$ Test de Muestras Independientes. Elaboración propia.

Fuente: Instituto Nacional de Estadística e Informática (2015).

En el primer factor, denominado «Familiar», tanto en el cluster 1 como en el 2 las medias son estadísticamente iguales; o sea, las regiones mineras no tienen ningún impacto en la reducción/aumento de la vulnerabilidad familiar. Asimismo, en el factor «Físico», los dos clúster obtuvieron medias estadísticamente iguales, indicando que las regiones mineras no tienen impacto en la vulnerabilidad física. En el tercer factor, «Social», tanto en el cluster 1 como en el cluster 2 las medias no son estadísticamente iguales, lo que significa que en las regiones mineras se tiene un impacto en la vulnerabilidad social. Los resultados de este estudio están de acuerdo con el trabajo de Carrere (2004) y Macdonald y Rowland (2002), que afirman que las actividades mineras tienen impacto negativo sobre la vulnerabilidad en ciertas comunidades. 


\section{ConClusiones}

Este estudio evalúa los impactos de las regiones mineras sobre la vulnerabilidad social. Los resultados permiten concluir que las regiones mineras no tienen presencia significativa sobre la vulnerabilidad física, compuesta por casos de feminicidios y violencia sexual a menores de 18 ańos; así como no afecta la vulnerabilidad familiar, que incluye las siguientes variables: las personas que fueron intervenidas por el consumo de drogas, denuncias por accidentes de tráfico, denuncias por delitos, violencia familiar y accidentes de tráfico no fatales.

Por otro lado, los departamentos considerados regiones mineras tienen mayor impacto sobre la vulnerabilidad social, que se compone de las variables: denuncias por faltas y robo de vehículos. El departamento de Madre Dios tiene poca población pero en los últimos ańos se ha generado mucha violencia. Los factores que explican estos índices son diversos, pero los principales son el desarrollo de la minería ilegal y el difícil control de zonas inaccesibles.

En este sentido, se recomienda estudiar más a fondo el impacto de las regiones mineras sobre la vulnerabilidad social, considerando que la minería implica un mayor crecimiento económico, lo que puede atraer empleo, pero también cambios en los estilos de vida de las familias, con un coste: el aumento de la vulnerabilidad social.

\section{Bibliografía}

Abramovay, M. y Pinheiro, C. L. (2003). Violência e vulnerabilidade social. En A. Fraerman (ed.), Inclusión social y desarrollo: Presente y futuro de la comunidad Iberoamericana (pp. 1-9). Madrid, España: Comunica.

Arellano, J. (2011). ¿Minería sin fronteras? Conflicto y desarrollo en regiones mineras del Perú. Lima, Perú: PUCP/UARM/IEP.

Barbetta, A. P. (2012). Estatística aplicada as Ciências Sociais. 8 ed. Florianópolis/SC, Brasil: Editora Ufsc.

Barrantes, R., Zárate, P. y Durand, A. (2005). Te quiero, pero no: relaciones entre minería, desarrollo y poblaciones locales. Lima, Perú: IEP/OXFAM.

Bebbington, A. y Humphreys, D. (2009). Actores y ambientalismos: conflictos socio-ambientales en Perú. Íconos. Revista de Ciencias Sociales, 15, 117-128.

Busso, G. (2001). Vulnerabilidad social: nociones e implicancias de políticas para Latinoamérica a inicios del siglo XXI. En Seminario Internacional: Las Diferentes expresiones de la Vulnerabilidad Social en América Latina y el Caribe. Seminario llevado a cabo en Santiago de Chile, Chile.

Carrere, R. (2004) (coord.). Minería, Impactos sociales y ambientales. Montevideo: Movimiento Mundial por los Bosques Tropicales. Montevideo, Uruguay: Editorial I. Rosgal S.A.

Contreras, C. (1988). Mineros y campesinos en los Andes. Lima, Perú: IEP.

Cunha, J. M. P. da (2004). Um sentido para a vulnerabilidade sociodemográfica nas metrópoles paulistas. Revista Brasileira de Estudos de População, 21(2), 343-347. 
De Echave, J., Diez, A., Huber, L., Revesz, B., Lanata, R. X. y Tanaka, M. (2009). Minería y conflicto social. Lima, Perú: IEP/CIPCA/CBC/CIES.

Duran, C. M. (2014). Informe Sectorial-Perú: Sector minero. Lima, Perú: Pacific Credit Rating.

Glave, M. y Kuramoto, J. (2007). La minería peruana: lo que sabemos y lo que aún nos falta por saber. En GRADE, Investigación, politicas y desarrollo en el Perú (pp. 135-181). Lima, Perú: Grade.

Hair, J. F., Black, W. C., Babin, B. J., Anderson, R. E. y Tatham, R. L. (2009). Análise Multivariada de Dados. $\sigma^{a}$ edição. Porto Alegre, Brasil: Bookman.

Instituto Nacional de Estadística e Informática (2015). Producto Bruto Interno por departamentos 2014. Lima, Perú: INEI.

Kaztman, R. (2000). Notas sobre la medición de la vulnerabilidad social. Santiago de Chile, Chile: BID/BIRF/CEPAL.

León, J. C. (2006). Vulnerability-A Conceptual and Methodological Review. Germany: Publication Series of UNU-EHS, 4. Bonn, Germany: Editorial: UNU-EHS.

Lira, A. D. y Aristondo, F. M. (2007). Panorama de la minería en el Perú. Lima, Perú: OSINERGMIN.

Loayza, N., Mier y Teran, A. y Rigolini, J. (2013). Poverty, Inequality, and the Local Natural Resource Curse. doi: 10.1596/1813-9450- 6366

Macdonald, I. y Rowland, C. (2002). Tunnel Vision: Women, mining and communities, Forum Report. Victoria, Australia: OXFAM Community Aid Abroad.

Macroconsult (2012). Impacto Económico de la Minería en el Perú. Lima, Perú: Sociedad Nacional de Minería, Petróleo y Energía.

Manteiga, G. M. T. y Vargas, P. A. (2009). Estadistica Aplicada: Una visión instrumental. Madrid, Espańa: Editorial Diaz de Santos.

Mendes, M. J. y Tavares, O. A. (2011). Risco, vulnerabilidade social e cidadania. Revista Crítica de Ciências Sociais, 93, 5-8.

Méndez, E., Orihuela, J. C. y Paredes, M. (2007). Minería y economía de los hogares en la sierra peruana: Impactos y espacios de conflicto. Lima, Perú: GRADE/CIES.

Ministerio de Energía y Minas (2015). Anuario Minero 2014: Reporte Estadístico. Lima, Perú: Editorial Ministerio de Energía y Minas.

Orihuela, J. C., Huaroto, C y Paredes, M. (2014). Escapando de la maldición de los recursos local: Conflictos socioambientales y salidas institucionales. Lima, Perú: CIES/PUCP.

Pizarro H. R. (2001). La vulnerabilidad social y sus desafíos: una mirada desde América Latina. Santiago de Chile, Chile: CEPAL.

Rodríguez, M. y Mora, R. (2001). Estadística informática: casos y ejemplos con el SPSS. Alicante, España: Publicaciones Universidad de Alicante.

Torralba, F. (1998). Antropología del cuidar. Madrid, España: Instituto Borja de Bioética y Fundación Mapfre Medicina.

Zegarra, E., Orihuela, J. C. y Paredes, M. (2007). Minería y economía de los hogares en la sierra: impactos y espacios de conflicto. Lima, Perú: GRADE. 